\title{
A viscosity hybrid steepest-descent method for a system of equilibrium and fixed point problems for an infinite family of strictly pseudo-contractive mappings
}

\author{
Uamporn Witthayarat ${ }^{1}$, Jong Kyu Kim² ${ }^{2}$ and Poom Kumam ${ }^{1 *}$
}

\section{"Correspondence: \\ poom.kum@kmutt.ac.th \\ ${ }^{1}$ Department of Mathematics, \\ Faculty of Science, King Mongkut's University of Technology Thonburi, KMUTT, Bangkok, 10140, Thailand Full list of author information is available at the end of the article}

\begin{abstract}
Based on a viscosity hybrid steepest-descent method, in this paper, we introduce an iterative scheme for finding a common element of a system of equilibrium and fixed point problems of an infinite family of strictly pseudo-contractive mappings which solves the variational inequality $\langle(\gamma f-\mu F) q, p-q\rangle \leq 0$ for $p \in \bigcap_{i=1}^{\infty} F\left(T_{i}\right)$. Furthermore, we also prove the strong convergence theorems for the proposed iterative scheme and give a numerical example to support and illustrate our main theorem.
\end{abstract}

MSC: 46C05; 47D03; 47H05,47H09; 47H10; 47H2O

Keywords: common fixed point; equilibrium problem; hybrid steepest-descent method; iterative algorithm; nonexpansive mapping; variational inequality

\section{Introduction}

Throughout this paper, we assume that $H$ is a real Hilbert space with inner product $\langle\cdot, \cdot\rangle$ and norm $\|\cdot\|$. Let $C$ be a nonempty closed convex subset of $H$. A self-mapping $f: C \rightarrow C$ is said to be a contraction on $C$ if there exists a constant $\alpha \in(0,1)$ such that $\|f(x)-f(y)\| \leq$ $\alpha\|x-y\|, \forall x, y \in C$. We denote by $\Pi_{C}$ the collection of mappings $f$ verifying the above inequality and note that each $f \in \Pi_{C}$ has a unique fixed point in $C$.

A mapping $T: C \rightarrow C$ is said to be $\lambda$-strictly pseudo-contractive if there exists a constant $\lambda \in[0,1)$ such that

$$
\|T x-T y\|^{2} \leq\|x-y\|^{2}+\lambda\|(I-T) x-(I-T) y\|^{2}, \quad \forall x, y \in C,
$$

and we denote by $F(T)$ the set of fixed points of the mapping T; that is, $F(T)=\{x \in C$ : $T x=x\}$.

Note that $T$ is the class of $\lambda$-strictly pseudo-contractive mappings including the class of nonexpansive mappings $T$ on $C$ (that is, $\|T x-T y\| \leq\|x-y\|, x, y \in C$ ) as a subclass. That is, $T$ is nonexpansive if and only if $T$ is 0 -strictly pseudo-contractive.

A mapping $F: C \rightarrow C$ is called $k$-Lipschitzian if there exists a positive constant $k$ such that

$$
\|F x-F y\| \leq k\|x-y\|, \quad \forall x, y \in C .
$$

\section{Springer}

(0) 2012 Witthayarat et al.; licensee Springer. This is an Open Access article distributed under the terms of the Creative Commons Attribution License (http://creativecommons.org/licenses/by/2.0), which permits unrestricted use, distribution, and reproduction in any medium, provided the original work is properly cited. 
$F$ is said to be $\eta$-strongly monotone if there exists a positive constant $\eta$ such that

$$
\langle F x-F y, x-y\rangle \geq \eta\|x-y\|^{2}, \quad \forall x, y \in C .
$$

Definition 1.1 A bounded linear operator $A$ is said to be strongly positive, if there exists a constant $\bar{\gamma}>0$ such that

$$
\langle A x, x\rangle \geq \bar{\gamma}\|x\|^{2}, \quad \forall x \in H
$$

In 2006, Marino and Xu [1] introduced the following iterative scheme: for $x_{1}=x \in C$,

$$
x_{n+1}=\alpha_{n} \gamma f\left(x_{n}\right)+\left(I-\alpha_{n} A\right) T x_{n}, \quad n \geq 1 .
$$

They proved that under appropriate conditions of the sequence $\left\{\alpha_{n}\right\}$, the sequence $\left\{x_{n}\right\}$ generated by (1.4) converges strongly to the unique solution of the variational inequality $\langle(\gamma f-A) q, p-q\rangle \leq 0, p \in F(T)$, which is the optimality condition for the minimization problem

$$
\min _{x \in C} \frac{1}{2}\langle A x, x\rangle-h(x)
$$

where $h$ is a potential function for $\gamma f$ (i.e., $h^{\prime}(x)=\gamma f(x)$ for $x \in H$ ).

In 2010, Jung [2] extended the result of Marino and $\mathrm{Xu}$ [1] to the class of $k$-strictly pseudo-contractive mappings $T: C \rightarrow H$ with $F(T) \neq \emptyset$ and introduced the following iterative scheme: for $x_{1}=x \in C$,

$$
\left\{\begin{array}{l}
y_{n}=\beta_{n} x_{n}+\left(1-\beta_{n}\right) P_{C} S x_{n}, \\
x_{n+1}=\alpha_{n} \gamma f\left(x_{n}\right)+\left(I-\alpha_{n} A\right) y_{n}, \quad n \geq 1,
\end{array}\right.
$$

where $S: C \rightarrow H$ is a mapping defined by $S x=k x+(1-k) T x$. He proved that the sequence $\left\{x_{n}\right\}$ generated by (1.5) converges strongly to a fixed point $q$ of $T$, which is the unique solution of the variational inequality

$$
\langle\gamma f(q)-A q, p-q\rangle \leq 0, \quad p \in F(T)
$$

Later, Tian [3] considered the following iterative method for a nonexpansive mapping $T: H \rightarrow H$ with $F(T) \neq \emptyset$,

$$
x_{n+1}=\alpha_{n} \gamma f\left(x_{n}\right)+\left(I-\mu \alpha_{n} F\right) T x_{n}, \quad n \geq 1,
$$

where $F$ is a $k$-Lipschitzian and $\eta$-strongly monotone operator. He proved that the sequence $\left\{x_{n}\right\}$ generated by (1.6) converges to a fixed point $q$ in $F(T)$, which is the unique solution of the variational inequality

$$
\langle(\gamma f-\mu F) q, p-q\rangle \leq 0, \quad p \in F(T)
$$

In 2010, Saeidi [4] introduced the following modified hybrid steepest-descent iterative algorithm for finding a common element of the set of solutions of a system of equilibrium 
problems for a family $\mathcal{F}=\left\{F_{j}: C \times C \rightarrow \mathbb{R}, j=1,2, \ldots, M\right\}$ and the set of common fixed points for a family of infinitely nonexpansive mappings $\mathcal{S}=\left\{S_{i}: C \rightarrow C\right\}$ with respect to $W$-mappings (see [5]):

$$
\left\{\begin{array}{l}
y_{n}=W_{n} J_{r_{M}, n}^{F_{M}} \cdots J_{r_{2}, n}^{F_{2}} J_{r_{1}, n}^{F_{1}} x_{n}, \\
x_{n+1}=\beta x_{n}+(1-\beta)\left(I-\lambda_{n} B\right) y_{n}, \quad \forall n \in \mathbb{N},
\end{array}\right.
$$

where $B$ is a relaxed $(\gamma, r)$-cocoercive, $k$-Lipschitzian mapping such that $r>\gamma k^{2}$. Then, under weaker hypotheses on coefficients, he proved the strongly convergence of the proposed iterative algorithm to the unique solution of the variational inequality.

Recently, Wang [6] extended and improved all the above results. He introduced a new iterative scheme: for $x_{1}=x \in C$,

$$
\left\{\begin{array}{l}
y_{n}=\beta_{n} x_{n}+\left(1-\beta_{n}\right) W_{n} x_{n}, \\
x_{n+1}=\alpha_{n} \gamma f\left(x_{n}\right)+\left(I-\mu \alpha_{n} F\right) y_{n}, \quad n \geq 1,
\end{array}\right.
$$

where $W_{n}$ is a mapping defined by (2.3), and $F$ is a $k$-Lipschitzian and $\eta$-strongly monotone operator with $0<\mu<2 \eta / k^{2}$. He proved that the sequence $\left\{x_{n}\right\}$ generated by (1.7) converges strongly to a common fixed point of an infinite family of $\lambda_{i}$-strictly pseudo-contractive mappings, which is a unique solution of the variational inequality

$$
\langle(\gamma f-\mu F) q, p-q\rangle \leq 0, \quad p \in \bigcap_{i=1}^{\infty} F\left(T_{i}\right) .
$$

Very recently, He, Liu and Cho [7] introduced an explicit scheme which was defined by the following suitable sequence:

$$
z_{n+1}=\epsilon_{n} \gamma f\left(z_{n}\right)+\left(I-\epsilon_{n} A\right) W_{n} S_{r_{1, n}}^{1} S_{r_{2, n}}^{2} \cdots S_{r_{K, n}}^{K} z_{n}, \quad \forall n \in N .
$$

They generated $W_{n}$-mapping by $\left\{T_{i}\right\}$ and $\left\{\lambda_{n}\right\}$ where $\left\{T_{i}\right\}$ is a family of nonexpansive mappings from $H$ into itself. They found that if $\left\{r_{k, n}\right\}_{k=1}^{K},\left\{\epsilon_{n}\right\}$ and $\left\{\lambda_{n}\right\}$ satisfy appropriate conditions and $F:=\left(\bigcap_{k=1}^{K} \operatorname{SEP}\left(G_{K}\right)\right) \cap\left(\bigcap_{n \in N} F\left(T_{n}\right)\right) \neq \emptyset$, then $\left\{z_{n}\right\}$ converges strongly to $x^{\prime \prime} \in F$, which satisfies the variational inequality $\left\langle(A-\gamma f) x^{\prime \prime}, x-x^{\prime \prime}\right\rangle \geq 0$ for all $x \in F$.

In this paper, we introduce a new iterative scheme in a Hilbert space $H$ which is a mixed iterative scheme of (1.7) and (1.8). We prove that the sequence converges strongly to a common element of the set of solutions of the system of equilibrium problems and the set of common fixed points of an infinite family of strictly pseudo-contractive mappings by using a viscosity hybrid steepest-descent method. The results obtained in this paper improved and extended the above mentioned results and many others. Finally, we give a simple numerical example to support and illustrate our main theorem in the last part.

\section{Preliminaries}

Let $H$ be a real Hilbert space and $C$ be a nonempty closed convex subset of $H$. We have

$$
\|x-y\|^{2}=\|x\|^{2}+\|y\|^{2}-2\langle x, y\rangle, \quad \forall x, y \in H .
$$


Recall that the nearest projection $P_{C}$ from $H$ to $C$ assigns to each $x \in H$ the unique point $P_{C} x \in C$ satisfying the property

$$
\left\|x-P_{C} x\right\|=\min _{y \in C}\|x-y\| .
$$

We recall some lemmas which will be needed in the rest of this paper.

Lemma 2.1 In a Hilbert space $H$, the following inequality holds:

$$
\|x+y\|^{2} \leq\|x\|^{2}+2\langle y, x+y\rangle, \quad x, y \in H .
$$

Lemma 2.2 Let $B$ be a $k$-Lipschitzian and $\eta$-strongly monotone operator on a Hilbertspace $H$ with $k>0, \eta>0,0<\mu<2 \eta / k^{2}$ and $0<t<1$. Then $S=(I-t \mu B): H \rightarrow H$ is a contraction with a contractive coefficient $1-t \tau$ and $\tau=\frac{1}{2} \mu\left(2 \eta-\mu k^{2}\right)$.

Proof From (1.2), (1.3) and (2.1), we have

$$
\begin{aligned}
\|S x-S y\|^{2} & =\|(x-y)-t \mu(B x-B y)\|^{2} \\
& =\|x-y\|^{2}+t^{2} \mu^{2}\|B x-B y\|^{2}-2 \mu t\langle B x-B y, x-y\rangle \\
& \leq\|x-y\|^{2}+t^{2} \mu^{2} k^{2}\|x-y\|^{2}-2 \mu t \eta\|x-y\|^{2} \\
& =\left[1-t \mu\left(2 \eta-\mu k^{2}\right)\right]\|x-y\|^{2} \\
& \leq(1-t \tau)^{2}\|x-y\|^{2},
\end{aligned}
$$

where $\tau=\frac{1}{2} \mu\left(2 \eta-\mu k^{2}\right)$, and so, $\|S x-S y\| \leq(1-t \tau)\|x-y\|$.

Hence, $S$ is a contraction with a contractive coefficient $1-t \tau$.

Lemma 2.3 Let $H$ be a Hilbert space. For a given $z \in H$ and $u \in C$,

$$
u=P_{C} z \quad \Leftrightarrow \quad\langle u-z, v-u\rangle \geq 0, \quad \forall v \in C .
$$

Lemma 2.4 Let $H$ be a real Hilbert space. For $q$ which solves the variational inequality $\langle(\gamma f-\mu B) q, p-q\rangle \leq 0, f \in \Pi_{H}, p \in F(T)$, the following statement is true:

$$
\langle(\gamma f-\mu B) q, p-q\rangle \leq 0 \quad \Leftrightarrow \quad P_{\Theta}(I-\mu B+\gamma f) q=q,
$$

where $\Theta:=\left(\bigcap_{i=1}^{\infty} F\left(T_{i}\right)\right) \cap\left(\bigcap_{j=1}^{M} S E P\left(F_{j}\right)\right)$.

Proof From Lemma (2.3), it follows that

$$
\begin{aligned}
q=P_{\Theta}(I-\mu B+\gamma f) q & \Leftrightarrow\langle q-(I-\mu B+\gamma f) q, p-q\rangle \geq 0, \quad p \in \Theta, \\
& \Leftrightarrow\langle(\mu B-\gamma f) q, p-q\rangle \geq 0 \\
& \Leftrightarrow\langle(\gamma f-\mu B) q, p-q\rangle \leq 0 .
\end{aligned}
$$


Lemma 2.5 [8] Let $C$ be a closed convex subset of a Hilbert space $H$ and $T: C \rightarrow C$ be a nonexpansive mapping with $F(T) \neq \emptyset$; if the sequence $\left\{x_{n}\right\}$ weakly converges to $x$ and $(I-T) x_{n}$ converges strongly to $y$, then $(I-T) x=y$.

Lemma 2.6 [9] Let $\left\{x_{n}\right\}$ and $\left\{z_{n}\right\}$ be bounded sequences in a Banach space E and $\left\{\gamma_{n}\right\}$ be a sequence in $[0,1]$ which satisfies the following condition:

$$
0<\liminf _{n \rightarrow \infty} \gamma_{n} \leq \limsup _{n \rightarrow \infty} \gamma_{n}<1
$$

Suppose that $x_{n+1}=\gamma_{n} x_{n}+\left(1-\gamma_{n}\right) z_{n}, n \geq 0$ and $\limsup _{n \rightarrow \infty}\left(\left\|z_{n+1}-z_{n}\right\|-\left\|x_{n+1}-x_{n}\right\|\right) \leq 0$. Then $\lim _{n \rightarrow \infty}\left\|z_{n}-x_{n}\right\|=0$.

Lemma 2.7 $[10,11]$ Let $\left\{s_{n}\right\}$ be a sequence of non-negative real numbers satisfying

$$
s_{n+1} \leq\left(1-\lambda_{n}\right) s_{n}+\lambda_{n} \delta_{n}+\gamma_{n}, \quad n \geq 0,
$$

where $\left\{\lambda_{n}\right\},\left\{\delta_{n}\right\}$ and $\left\{\gamma_{n}\right\}$ satisfy the following conditions:

(i) $\left\{\lambda_{n}\right\} \subset[0,1]$ and $\sum_{n=0}^{\infty}=\infty$;

(ii) $\lim \sup _{n \rightarrow \infty} \delta_{n} \leq 0$ or $\sum_{n=0}^{\infty} \lambda_{n} \delta_{n}<\infty$;

(iii) $\gamma_{n} \geq 0(n \geq 0), \sum_{n=0}^{\infty} \gamma_{n}<\infty$.

Then $\lim _{n \rightarrow \infty} s_{n}=0$.

Lemma 2.8 [12] Let $C$ be a nonempty closed convex subset of a real Hilbert space $H$ and $T: C \rightarrow C$ be a $\lambda$-strictly pseudo-contractive mapping. Define a mapping $S: C \rightarrow C$ by $S x=\alpha x+(1-\alpha)$ Tx for all $x \in C$ and $\alpha \in[\lambda, 1)$. Then $S$ is a nonexpansive mapping such that $F(S)=F(T)$.

In this work, we defined the mapping $W_{n}$ by

$$
\begin{aligned}
& U_{n, n+1}=I, \\
& U_{n, n}=\gamma_{n} T_{n}^{\prime} U_{n, n+1}+\left(1-\gamma_{n}\right) I, \\
& U_{n, n-1}=\gamma_{n-1} T_{n-1}^{\prime} U_{n, n}+\left(1-\gamma_{n-1}\right) I, \\
& \vdots \\
& U_{n, k}=\gamma_{k} T_{k}^{\prime} U_{n, k+1}+\left(1-\gamma_{k}\right) I, \\
& U_{n, k-1}=\gamma_{k-1} T_{k-1}^{\prime} U_{n, k}+\left(1-\gamma_{k-1}\right) I, \\
& \vdots \\
& U_{n, 2}=\gamma_{2} T_{2}^{\prime} U_{n, 3}+\left(1-\gamma_{2}\right) I, \\
& W_{n}=U_{n, 1}=\gamma_{1} T_{1}^{\prime} U_{n, 2}+\left(1-\gamma_{1}\right) I,
\end{aligned}
$$

where $\gamma_{1}, \gamma_{2}, \ldots$ are real numbers such that $0 \leq \gamma_{n} \leq 1, T_{i}^{\prime}=\theta_{i} I+\left(1-\theta_{i}\right) T_{i}$ where $T_{i}$ is a $\lambda_{i}$-strictly pseudo-contractive mapping of $C$ into itself and $\theta_{i} \in\left[\lambda_{i}, 1\right)$. By Lemma 2.8 , we know that $T_{i}^{\prime}$ is a nonexpansive mapping and $F\left(T_{i}\right)=F\left(T_{i}^{\prime}\right)$. As a result, it can be easily seen that $W_{n}$ is also a nonexpansive mapping. 
Lemma 2.9 [5] Let $C$ be a nonempty closed convex subset of a strictly convex Banach space E. Let $T_{1}^{\prime}, T_{2}^{\prime}, \ldots$ be nonexpansive mappings of $C$ into itself such that $\bigcap_{i=1}^{\infty} F\left(T_{i}^{\prime}\right) \neq \emptyset$ and $\gamma_{1}, \gamma_{2}, \ldots$ be real numbers such that $0<\gamma_{i} \leq b<1$ for each $i=1,2, \ldots$. Then for any $x \in C$ and $k \in N$, the limit $\lim _{n \rightarrow \infty} U_{n, k} x$ exists.

By using Lemma 2.8, one can define the mapping $W$ of $C$ into itself as follows:

$$
W x:=\lim _{n \rightarrow \infty} W_{n} x=\lim _{n \rightarrow \infty} U_{n, 1} x, \quad x \in C .
$$

Such a mapping $W$ is called the modified $W$-mapping generated by $T_{1}, T_{2}, \ldots, \gamma_{1}, \gamma_{2}, \ldots$ and $\theta_{1}, \theta_{2}, \ldots$

Lemma 2.10 [5] Let $C$ be a nonempty closed convex subset of a strictly convex Banach space E. Let $T_{1}^{\prime}, T_{2}^{\prime}, \ldots$ be nonexpansive mappings of $C$ into itself such that $\bigcap_{i=1}^{\infty} F\left(T_{i}^{\prime}\right) \neq \emptyset$ and $\gamma_{1}, \gamma_{2}, \ldots$ be real numbers such that $0<\gamma_{i} \leq b<1$ for each $i=1,2, \ldots$. Then $F(W)=$ $\bigcap_{i=1}^{\infty} F\left(T_{i}^{\prime}\right)$.

Combining Lemmas 2.7-2.9, one can get that $F(W)=\bigcap_{i=1}^{\infty} F\left(T_{i}^{\prime}\right)=\bigcap_{i=1}^{\infty} F\left(T_{i}\right)$.

Lemma 2.11 [13] Let $C$ be a nonempty closed convex subset of a Hilbert space $H,\left\{T_{i}^{\prime}\right.$ : $C \rightarrow C\}$ be a family of infinite nonexpansive mappings with $\bigcap_{i=1}^{\infty} F\left(T_{i}^{\prime}\right) \neq \emptyset,\left\{\gamma_{i}\right\}$ be a real sequence such that $0<\gamma_{i} \leq b<1$, for each $i \geq 1$. If $K$ is any bounded subset of $C$, then

$$
\lim _{n \rightarrow \infty} \sup _{x \in K}\left\|W x-W_{n} x\right\|=0 .
$$

For solving the equilibrium problem, let us give the following assumptions on a bifunction $F: C \times C \rightarrow \mathbb{R}$, which were imposed in [14]:

(A1) $F(x, x)=0$ for all $x \in C$;

(A2) $F$ is monotone, i.e., $F(x, y)+F(y, x) \leq 0$ for all $x, y \in C$;

(A3) for each $x, y, z \in C, \lim _{t \downarrow 0} F(t z+(1-t) x, y) \leq F(x, y)$;

(A4) for each $x \in C, y \mapsto F(x, y)$ is convex and lower semicontinuous.

Lemma 2.12 [14] Let $C$ be a nonempty closed convex subset of $H$, and let $F$ be a bifunction of $C \times C$ into $\mathbb{R}$ satisfying (A1)-(A4). Then for $r>0$ and $x \in H$, there exists $z \in C$ such that

$$
F(z, y)+\frac{1}{r}\langle y-z, z-x\rangle \geq 0
$$

Lemma 2.13 [15] Let $C$ be a nonempty closed convex subset of $H$, and let $F$ be a bifunction of $C \times C$ into $\mathbb{R}$ satisfying (A1)-(A4). For $r>0$, define a mapping $J_{r}^{F}: H \rightarrow C$ as follows:

$$
J_{r}^{F}(x)=\left\{z \in C: F(z, y)+\frac{1}{r}\langle y-z, z-x\rangle \geq 0, \forall y \in C\right\}
$$

for all $x \in H$. Then the following conclusions hold:

(1) $J_{r}^{F}$ is single-valued;

(2) $J_{r}^{F}$ is firmly nonexpansive, i.e., for any $x, y \in H$,

$$
\left\|J_{r}^{F}(x)-J_{r}^{F}(y)\right\|^{2} \leq\left\langle J_{r}^{F}(x)-J_{r}^{F}(y), x-y\right\rangle ;
$$


(3) $F\left(J_{r}^{F}\right)=E P(F)$;

(4) $E P(F)$ is closed and convex.

Lemma 2.14 [5] Let $C$ be a nonempty closed convex subset of a real Hilbert space H. Let $\mathcal{T}=\left\{T_{i}\right\}_{i=1}^{N}$ be an infinite family of nonexpanxive mappings with $F(\mathcal{T})=\bigcap_{i=1}^{\infty} F\left(T_{i}\right) \neq \emptyset$ and $\left\{\gamma_{i}\right\}$ be a real sequence such that $0<\gamma_{i} \leq b<1$ for each $i \geq 1$. Then:

(1) $W_{n}$ is nonexpansive and $F\left(W_{n}\right)=\bigcap_{i=1}^{n} F\left(T_{i}\right)$ for each $n \geq 1$;

(2) for each $x \in C$ and for each positive integer $k$, the limit $\lim _{n \rightarrow \infty} U_{n, k} x$ exists;

(3) the mapping $W: C \rightarrow C$ defined by $W x=\lim _{n \rightarrow \infty} W_{n} x=\lim _{n \rightarrow \infty} U_{n, 1} x$ is a nonexpansive mapping satisfying $F(W)=F(\mathcal{T})$ and it is called the $W$-mapping generated by $T_{1}, T_{2}, \ldots$ and $\gamma_{1}, \gamma_{2}, \ldots$;

(4) if $K$ is any bounded subset of $C$, then $\lim _{n \rightarrow \infty} \sup _{x \in K}\left\|W x-W_{n} x\right\|=0$.

\section{Main results}

In this section, we will introduce an iterative scheme by using a viscosity hybrid steepestdescent method for finding a common element of the set of variational inequalities, fixed points for an infinite family of strictly pseudo-contractive mappings and the set of solutions of a system of equilibrium problems in a real Hilbert space.

Theorem 3.1 Let $C$ be a nonempty closed convex subset of a real Hilbert space H, let $T_{i}: H \rightarrow H$ be a $\lambda_{i}$-strictly pseudo-contractive mapping with $\bigcap_{i=1}^{\infty} F\left(T_{i}\right) \neq \emptyset, \mathcal{F}=\left\{F_{j}: j=\right.$ $1,2,3, \ldots, M\}$ be a finite family of bifunctions $C \times C$ into $\mathbb{R}$ satisfying (A1)-(A4) and $\gamma_{i}$ be a real sequence such that $0 \leq \gamma_{i} \leq b \leq 1$ for each $i \geq 1$. Let $B$ be a $k$-Lipschitzian and $\eta$ strongly monotone operator on $C$ with $0<\mu<\eta / k^{2}$ and $f \in \Pi_{H}$ with $0<\gamma<\mu\left(\eta-\frac{\mu k^{2}}{2}\right) / \alpha=$ $\tau / \alpha$ and $\tau<1$. Assume that $\Theta:=\left(\bigcap_{i=1}^{\infty} F\left(T_{i}\right)\right) \cap\left(\bigcap_{j=1}^{M} S E P\left(F_{j}\right)\right) \neq \emptyset$. Let the mapping $W_{n}$ be defined by (2.3). Let $\left\{x_{n}\right\}$ be the sequence generated by $x_{1} \in H$ and

$$
\left\{\begin{array}{l}
u_{n}=J_{r_{M, n}}^{F_{M}} J_{r_{M-1, n}}^{F_{M-1}} \cdots J_{r_{2, n}}^{F_{2}} J_{r_{1, N}}^{F_{1}} x_{n}, \\
y_{n}=\beta_{n} x_{n}+\left(1-\beta_{n}\right) W_{n} u_{n}, \\
x_{n+1}=\alpha_{n} \gamma f\left(x_{n}\right)+\left(I-\alpha_{n} \mu B\right) y_{n}, \quad \forall n \in \mathbb{N} \text { and } n \geq 1,
\end{array}\right.
$$

where $\left\{\alpha_{n}\right\}$ and $\left\{\beta_{n}\right\}$ are sequences in $(0,1)$ which satisfy the following conditions:

(C1) $\lim _{n \rightarrow \infty} \alpha_{n}=0$ and $\Sigma_{n=1}^{\infty} \alpha_{n}=\infty$;

(C2) $0<\liminf _{n \rightarrow \infty} \beta_{n}<\limsup _{n \rightarrow \infty} \beta_{n} \leq a<1$ for some constant $a \in(0,1)$;

(C3) $\liminf _{n \rightarrow \infty} r_{j, n}>0$, for each $j=1,2, \ldots, M$.

Then the sequence $\left\{x_{n}\right\}$ converges strongly to $q \in \Theta$, where $q=P_{\Theta}(I-\mu B+\gamma f) q$, which is the unique solution of the variational inequality

$$
\langle(\gamma f-\mu B) q, p-q\rangle \leq 0, \quad \forall p \in \Theta
$$

or equivalently, $q$ is the unique solution of the minimization problem

$$
\min _{x \in \Theta} \frac{1}{2}\langle A x, x\rangle+h(x)
$$

where $h$ is a potential function for $\gamma f$. 
Proof We will divide the proof of Theorem 3.1 into several steps.

Step 1. We show that $\left\{x_{n}\right\}$ is bounded. Let $p \in \Theta$. Since for each $k=1,2,3, \ldots, M, J_{r_{k, n}}^{F_{k}}$ is nonexpansive. Given $\Im_{n}^{k}=J_{r_{k, n}}^{F_{k}} J_{r_{k-1, n}}^{F_{k-1}} J_{r_{k-2, n}}^{F_{k-2}} \ldots J_{r_{2, n}}^{F_{2}} J_{r_{1, n}}^{F_{1}}$ for $k \in\{1,2,3, \ldots, M\}$ and $\Im_{n}^{0}=I$, for each $n \in \mathbb{N}$, we have

$$
\left\|\Im_{n}^{k} x_{n}-p\right\|=\left\|\Im_{n}^{k} x_{n}-\Im_{n}^{k} p\right\| \leq\left\|x_{n}-p\right\| .
$$

Consider,

$$
\begin{aligned}
\left\|y_{n}-p\right\| & =\left\|\beta_{n} x_{n}+\left(1-\beta_{n}\right) W_{n} u_{n}-p\right\| \\
& =\left\|\beta_{n}\left(x_{n}-p\right)+\left(1-\beta_{n}\right)\left(W_{n} u_{n}-p\right)\right\| \\
& \leq \beta_{n}\left\|x_{n}-p\right\|+\left(1-\beta_{n}\right)\left\|\left(W_{n} u_{n}-p\right)\right\| \\
& =\beta_{n}\left\|x_{n}-p\right\|+\left(1-\beta_{n}\right)\left\|W_{n} \Im_{n}^{M} x_{n}-p\right\| \\
& \leq \beta_{n}\left\|x_{n}-p\right\|+\left(1-\beta_{n}\right)\left\|\Im_{\Im_{n}}^{M} x_{n}-p\right\| \\
& \leq \beta_{n}\left\|x_{n}-p\right\|+\left(1-\beta_{n}\right)\left\|x_{n}-p\right\| \\
& =\left\|x_{n}-p\right\| .
\end{aligned}
$$

From Lemma 2.2, (3.1) and (3.3), it follows that

$$
\begin{aligned}
\left\|x_{n+1}-p\right\| & =\left\|\alpha_{n} \gamma f\left(x_{n}\right)+\left(I-\alpha_{n} \mu B\right) y_{n}-p\right\| \\
& =\left\|\alpha_{n}\left(\gamma f\left(x_{n}\right)-\mu B p\right)+\left(I-\alpha_{n} \mu B\right) y_{n}-\left(I-\alpha_{n} \mu B\right) p\right\| \\
& =\left\|\left(I-\alpha_{n} \mu B\right)\left(y_{n}-p\right)+\alpha_{n}\left(\gamma f\left(x_{n}\right)-\mu B p+\gamma f(p)-\gamma f(p)\right)\right\| \\
& \leq\left\|\left(I-\alpha_{n} \mu B\right)\left(y_{n}-p\right)\right\|+\alpha_{n} \gamma\left\|f\left(x_{n}\right)-f(p)\right\|+\alpha_{n}\|\gamma f(p)-\mu B p\| \\
& \leq\left(1-\alpha_{n} \tau\right)\left\|y_{n}-p\right\|+\alpha_{n} \gamma \alpha\left\|x_{n}-p\right\|+\alpha_{n}\|\gamma f(p)-\mu B p\| \\
& \leq\left(1-\alpha_{n} \tau\right)\left\|x_{n}-p\right\|+\alpha_{n} \gamma \alpha\left\|x_{n}-p\right\|+\alpha_{n}\|\gamma f(p)-\mu B p\| \\
& =\left[1-\alpha_{n}(\tau-\gamma \alpha)\right]\left\|x_{n}-p\right\|+\alpha_{n} \frac{\tau-\gamma \alpha}{\tau-\gamma \alpha}\|\gamma f(p)-\mu B p\| \\
& \leq \max \left\{\left\|x_{n}-p\right\|, \frac{\|\gamma f(p)-\mu B p\|}{\tau-\gamma \alpha}\right\} ; \quad n \geq 1 .
\end{aligned}
$$

By mathematical induction, we have

$$
\left\|x_{n}-p\right\| \leq \max \left\{\left\|x_{1}-p\right\|, \frac{\|\gamma f(p)-\mu B p\|}{\tau-\gamma \alpha}\right\}, \quad \forall n \geq 1,
$$

and we obtain $\left\{x_{n}\right\}$ is bounded. So are $\left\{y_{n}\right\},\left\{W_{n} \Im_{n}^{k}\left(x_{n}\right)\right\}$ and $\left\{f\left(x_{n}\right)\right\}$.

Step 2. We claim that if $\left\{x_{n}\right\}$ is a bounded sequence in $C$, then

$$
\lim _{n \rightarrow \infty}\left\|\Im_{n}^{k} x_{n}-\Im_{n+1}^{k} x_{n}\right\|=0
$$

for every $k \in\{1,2,3, \ldots, M\}$. From Step 2 of the proof in [16, Theorem 3.1], we have for $k \in\{1,2,3, \ldots, M\}$,

$$
\lim _{n \rightarrow \infty}\left\|J_{r_{k, n+1}}^{F_{k}} x_{n}-J_{r_{k, n}}^{F_{k}} x_{n}\right\|=0
$$


Note that for every $k \in\{1,2,3, \ldots, M\}$, we have

$$
\Im_{n}^{k}=J_{r_{k, n}}^{F_{k}} J_{r_{k-1, n}}^{F_{k-1}} J_{r_{k-2, n}}^{F_{k-2}} \cdots J_{r_{2, n}}^{F_{2}} J_{r_{1, n}}^{F_{1}}=J_{r_{k, n}}^{F_{k}} \Im_{n}^{k-1}
$$

So, we note that

$$
\begin{aligned}
\left\|\Im_{n}^{k} x_{n}-\Im_{n+1}^{k} x_{n}\right\|= & \left\|J_{r_{k, n}}^{F_{k}} \Im_{n}^{k-1} x_{n}-J_{r_{k, n+1}}^{F_{k}} \Im_{n+1}^{k-1} x_{n}\right\| \\
\leq & \left\|J_{r_{k, n}}^{F_{k}} \Im_{n}^{k-1} x_{n}-J_{r_{k, n+1}}^{F_{k}} \Im_{n}^{k-1} x_{n}\right\|+\left\|J_{r_{k, n+1}}^{F_{k}} \Im_{n}^{k-1} x_{n}-J_{r_{k, n+1}}^{F_{k}} \Im_{n+1}^{k-1} x_{n}\right\| \\
\leq & \left\|J_{r_{k, n}}^{F_{k}} \Im_{n}^{k-1} x_{n}-J_{r_{k, n+1}}^{F_{k}} \Im_{n}^{k-1} x_{n}\right\|+\left\|\Im_{n}^{k-1} x_{n}-\Im_{n+1}^{k-1} x_{n}\right\| \\
\leq & \left\|J_{r_{k, n}}^{F_{k}} \Im_{n}^{k-1} x_{n}-J_{r_{k, n+1}}^{F_{k}} \Im_{n}^{k-1} x_{n}\right\|+\left\|J_{r_{k-1, n}}^{F_{k-1}} \Im_{n}^{k-2} x_{n}-J_{r_{k-1, n+1}}^{F_{k-1}} \Im_{n}^{k-2} x_{n}\right\| \\
& +\left\|\Im_{n}^{k-2} x_{n}-\Im_{n+1}^{k-2} x_{n}\right\| \\
\leq & \left\|J_{r_{k, n}}^{F_{k}} \Im_{n}^{k-1} x_{n}-J_{r_{k, n+1}}^{F_{k}} \Im_{n}^{k-1} x_{n}\right\|+\left\|J_{r_{k-1, n}}^{F_{k-1}} \Im_{n}^{k-2} x_{n}-J_{r_{k-1, n+1}}^{F_{k-1}} \Im_{n}^{k-2} x_{n}\right\| \\
& +\cdots+\left\|J_{r_{2, n}}^{F_{2}} \Im_{n}^{1} x_{n}-J_{r_{2, n+1}}^{F_{2}} \Im_{n}^{1} x_{n}\right\|+\left\|J_{r_{1, n}}^{F_{1}} x_{n}-J_{r_{1, n+1}}^{F_{1}} x_{n}\right\| .
\end{aligned}
$$

Now, applying (3.7) to (3.8), we conclude (3.6).

Step 3. We show that $\lim _{n \rightarrow \infty}\left\|x_{n+1}-x_{n}\right\|=0$.

We define a sequence $\left\{z_{n}\right\}$ by $z_{n}=\left(x_{n+1}-\beta_{n} x_{n}\right) /\left(1-\beta_{n}\right)$, so that $x_{n+1}=\beta_{n} x_{n}+\left(1-\beta_{n}\right) z_{n}$. We now observe that

$$
\begin{aligned}
z_{n+1}-z_{n}= & \frac{x_{n+2}-\beta_{n+1} x_{n+1}}{1-\beta_{n+1}}-\frac{x_{n+1}-\beta_{n} x_{n}}{1-\beta_{n}} \\
= & \frac{\alpha_{n+1} \gamma f\left(x_{n+1}\right)+\left(I-\mu \alpha_{n+1} B\right) y_{n+1}-\beta_{n+1} x_{n+1}}{1-\beta_{n+1}} \\
& -\frac{\alpha_{n} \gamma f\left(x_{n}\right)+\left(I-\mu \alpha_{n} B\right) y_{n}-\beta_{n} x_{n}}{1-\beta_{n}} \\
= & \frac{\alpha_{n+1}\left(\gamma f\left(x_{n+1}\right)-\mu B y_{n+1}\right)}{1-\beta_{n+1}}-\frac{\alpha_{n}\left(\gamma f\left(x_{n}\right)-\mu B y_{n}\right)}{1-\beta_{n}}+W_{n+1} u_{n+1}-W_{n} u_{n} .
\end{aligned}
$$

It follows from (3.9) that

$$
\begin{aligned}
\left\|z_{n+1}-z_{n}\right\| \leq & \frac{\alpha_{n+1}}{1-\beta_{n+1}}\left(\left\|\gamma f\left(x_{n+1}\right)\right\|+\left\|\mu B y_{n+1}\right\|\right)+\frac{\alpha_{n}}{1-\beta_{n}}\left(\left\|\gamma f\left(x_{n}\right)\right\|+\left\|\mu B y_{n}\right\|\right) \\
& +\left\|W_{n+1} u_{n+1}-W_{n} u_{n}\right\| .
\end{aligned}
$$

We observe that

$$
\begin{aligned}
& \left\|W_{n+1} u_{n+1}-W_{n} u_{n}\right\| \\
& \quad=\left\|W_{n+1} \Im_{n+1}^{k} x_{n+1}-W_{n} \Im_{n}^{k} x_{n}\right\| \\
& \quad \leq\left\|W_{n+1} \Im_{n+1}^{k} x_{n+1}-W_{n+1} \Im_{n} x_{n}\right\|+\left\|W_{n+1} \Im_{n}^{k} x_{n+1}-W_{n} \Im_{n}^{k} x_{n}\right\|,
\end{aligned}
$$

and compute

$$
\begin{aligned}
\left\|W_{n+1} \Im_{n}^{k} x_{n+1}-W_{n} \Im_{n}^{k} x_{n}\right\| & \leq\left\|W_{n+1} \Im_{n}^{k} x_{n+1}-W_{n+1} \Im_{n}^{k} x_{n}\right\|+\left\|W_{n+1} \Im_{n}^{k} x_{n}-W_{n} \Im_{n}^{k} x_{n}\right\| \\
& \leq\left\|x_{n+1}-x_{n}\right\|+\left\|W_{n+1} \Im_{n}^{k} x_{n}-W_{n} \Im_{n}^{k} x_{n}\right\| .
\end{aligned}
$$


Consider,

$$
\begin{aligned}
\left\|W_{n+1} \Im_{n}^{k} x_{n}-W_{n} \Im_{n}^{k} x_{n}\right\| & =\left\|\gamma_{1} T_{1}^{\prime} U_{n+1,2} \Im_{n}^{k} x_{n}-\gamma_{1} T_{1}^{\prime} U_{n, 2} \Im_{n}^{k} x_{n}\right\| \\
& \leq \gamma_{1}\left\|U_{n+1,2} \Im_{n}^{k} x_{n}-U_{n, 2} \Im_{n}^{k} x_{n}\right\| \\
& =\gamma_{1}\left\|\gamma_{2} T_{2}^{\prime} U_{n+1,3} \Im_{n}^{k} x_{n}-\gamma_{2} T_{2}^{\prime} U_{n, 3} \Im_{n}^{k} x_{n}\right\| \\
& \leq \cdots \\
& \leq \gamma_{1} \gamma_{2} \cdots \gamma_{n}\left\|U_{n+1, n+1} \Im_{n}^{k} x_{n}-U_{n, n+1} \Im_{n}^{k} x_{n}\right\| \\
& \leq M_{1} \prod_{i=1}^{n} \gamma_{i},
\end{aligned}
$$

where $M_{1} \geq 0$ is a constant such that $\left\|U_{n+1, n+1} u_{n}-U_{n, n+1} u_{n}\right\| \leq M_{1}$ for all $n \geq 1$.

Substituting (3.11) and (3.13) into (3.10), we can obtain

$$
\begin{aligned}
\left\|z_{n+1}-z_{n}\right\| \leq & \frac{\alpha_{n+1}}{1-\beta_{n+1}}\left(\left\|\gamma f\left(x_{n+1}\right)\right\|+\left\|\mu B y_{n+1}\right\|\right)+\frac{\alpha_{n}}{1-\beta_{n}}\left(\left\|\gamma f\left(x_{n}\right)\right\|+\left\|\mu B y_{n}\right\|\right) \\
& +\left\|W_{n+1} \Im_{n+1}^{k} x_{n+1}-W_{n+1} \Im_{n}^{k} x_{n+1}\right\|+\left\|x_{n+1}-x_{n}\right\|+M_{1} \prod_{i=1}^{n} \gamma_{i} \\
\leq & M_{2}\left(\frac{\alpha_{n+1}}{1-\beta_{n+1}}+\frac{\alpha_{n}}{1-\beta_{n}}\right)+\left\|W_{n+1} \Im_{n+1}^{k} x_{n+1}-W_{n+1} \Im_{n}^{k} x_{n+1}\right\| \\
& +\left\|x_{n+1}-x_{n}\right\|+M_{1} \prod_{i=1}^{n} \gamma_{i}
\end{aligned}
$$

where $M_{2}=\sup \left\{\left\|\gamma f\left(x_{n}\right)\right\|+\left\|\mu B y_{n}\right\|, n \geq 1\right\}$.

It follows from (3.14) that

$$
\begin{aligned}
\left\|z_{n+1}-z_{n}\right\| \leq & M_{2}\left(\frac{\alpha_{n+1}}{1-\beta_{n+1}}+\frac{\alpha_{n}}{1-\beta_{n}}\right)+\left\|\Im_{n+1}^{k} x_{n+1}-\Im_{n}^{k} x_{n+1}\right\| \\
& +\left\|x_{n+1}-x_{n}\right\|+M_{1} \prod_{i=1}^{n} \gamma_{i} .
\end{aligned}
$$

Hence, we have

$$
\left\|z_{n+1}-z_{n}\right\|-\left\|x_{n+1}-x_{n}\right\| \leq M_{2}\left(\frac{\alpha_{n+1}}{1-\beta_{n+1}}+\frac{\alpha_{n}}{1-\beta_{n}}\right)+\left\|\Im_{n+1}^{k} x_{n+1}-\Im_{n}^{k} x_{n+1}\right\|+M_{1} \prod_{i=1}^{n} \gamma_{i} .
$$

From $\lim _{n \rightarrow \infty}\left\|\Im_{n}^{k} x_{n}-\Im_{n+1}^{k} x_{n}\right\|=0$ and the condition $\lim _{n \rightarrow \infty} \alpha_{n}=0$ and $0<$ $\lim _{n \rightarrow \infty} \inf \beta_{n}<\lim _{n \rightarrow \infty} \sup \beta_{n} \leq a<1$ for some $a \in(0,1)$, it follows that

$$
\lim _{n \rightarrow \infty} \sup \left(\left\|z_{n+1}-z_{n}\right\|-\left\|x_{n+1}-x_{n}\right\|\right) \leq 0
$$

By Lemma 2.5 , we obtain

$$
\lim _{n \rightarrow \infty}\left\|z_{n}-x_{n}\right\|=0
$$


From $x_{n+1}=\beta_{n} x_{n}+\left(1-\beta_{n}\right) z_{n}$ and by (3.16), we get

$$
\left\|x_{n+1}-x_{n}\right\|=\left(1-\beta_{n}\right)\left\|z_{n}-x_{n}\right\| .
$$

Hence,

$$
\lim _{n \rightarrow \infty}\left\|x_{n+1}-x_{n}\right\|=\lim _{n \rightarrow \infty}\left(1-\beta_{n}\right)\left\|z_{n}-x_{n}\right\|=0
$$

Step 4. We claim that $\lim _{n \rightarrow \infty}\left\|x_{n}-W_{n} u_{n}\right\|=0$.

$$
\begin{aligned}
\left\|x_{n}-W_{n} u_{n}\right\| & \leq\left\|x_{n}-x_{n+1}\right\|+\left\|x_{n+1}-W_{n} u_{n}\right\| \\
& =\left\|x_{n}-x_{n+1}\right\|+\left\|\alpha_{n} \gamma f\left(x_{n}\right)+\left(I-\alpha_{n} \mu B\right) y_{n}-W_{n} u_{n}\right\| \\
& =\left\|x_{n}-x_{n+1}\right\|+\left\|\alpha_{n} \gamma f\left(x_{n}\right)+y_{n}-\alpha_{n} \mu B y_{n}-W_{n} u_{n}\right\| \\
& \leq\left\|x_{n+1}-x_{n}\right\|+\alpha_{n}\left\|\gamma f\left(x_{n}\right)-\mu B y_{n}\right\|+\left\|y_{n}-W_{n} u_{n}\right\| \\
& \leq\left\|x_{n+1}-x_{n}\right\|+\alpha_{n}\left\|\gamma f\left(x_{n}\right)-\mu B y_{n}\right\|+\left\|\beta_{n} x_{n}+\left(1-\beta_{n}\right) W_{n} u_{n}-W_{n} u_{n}\right\| \\
& \leq\left\|x_{n+1}-x_{n}\right\|+\alpha_{n}\left\|\gamma f\left(x_{n}\right)-\mu B y_{n}\right\|+\beta_{n}\left\|x_{n}-W_{n} u_{n}\right\| .
\end{aligned}
$$

It follows that

$$
\left\|x_{n}-W_{n} u_{n}\right\| \leq \frac{1}{1-\beta_{n}}\left\|x_{n}-x_{n+1}\right\|+\frac{\alpha_{n}}{1-\beta_{n}}\left\|\gamma f\left(x_{n}\right)-\mu B y_{n}\right\|
$$

By the conditions $(\mathrm{C} 1)$ and $(\mathrm{C} 2)$, we obtain

$$
\lim _{n \rightarrow \infty}\left\|x_{n}-W_{n} u_{n}\right\|=0
$$

Step 5. We show that

$$
\lim _{n \rightarrow \infty}\left\|\Im_{n^{k}}^{k} x_{n}-\Im_{n}^{k+1} x_{n}\right\|=0, \quad \forall k=1,2, \ldots, M-1
$$

for any $p \in \Theta$ and $\forall k=1,2, \ldots, M-1$. We note that $J_{r_{k+1, n}}^{F_{k+1}}$ is firmly nonexpansive by Lemma 2.12, then we observe that

$$
\begin{aligned}
\left\|\Im_{n}^{k+1} x_{n}-p\right\| & =\left\|J_{r_{k+1, n}}^{F_{k+1}} \Im_{n}^{k} x_{n}-J_{r_{k+1, n}}^{F_{k+1}} p\right\| \\
& \leq\left\langle J_{r_{k+1, n}}^{F_{k+1}} \Im_{n}^{k} x_{n}-J_{r_{k+1, n}}^{F_{k+1}} p, \Im_{n}^{k} x_{n}-p\right\rangle \\
& =\left\langle\Im_{n}^{k+1} x_{n}-p, \Im_{n}^{k} x_{n}-p\right\rangle \\
& =\frac{1}{2}\left(\left\|\Im_{n}^{k+1} x_{n}-p\right\|^{2}+\left\|\Im_{n}^{k} x_{n}-p\right\|^{2}-\left\|\Im_{n}^{k+1} x_{n}-\Im_{n}^{k} x_{n}\right\|^{2}\right),
\end{aligned}
$$

and hence

$$
\begin{aligned}
\left\|\Im_{n}^{k+1} x_{n}-p\right\|^{2} & \leq\left\|\Im_{n}^{k} x_{n}-p\right\|^{2}-\left\|\Im_{n}^{k+1} x_{n}-\Im_{n}^{k} x_{n}\right\|^{2} \\
& \leq\left\|x_{n}-p\right\|^{2}-\left\|\Im_{n}^{k+1} x_{n}-\Im_{n}^{k} x_{n}\right\|^{2} .
\end{aligned}
$$


It follows that

$$
\begin{aligned}
& \left\|x_{n+1}-p\right\|^{2}=\left\|\alpha_{n}\left(\gamma f\left(x_{n}\right)-\mu B p\right)+\left(I-\alpha_{n} \mu B\right)\left(y_{n}-p\right)\right\|^{2} \\
& =\alpha_{n}^{2}\left\|\left(\gamma f\left(x_{n}\right)-\mu B p\right)\right\|^{2}+\left(1-\alpha_{n} \tau\right)^{2}\left\|y_{n}-p\right\|^{2} \\
& +2 \alpha_{n}\left\{\left(\gamma f\left(x_{n}\right)-\mu B p\right),\left(I-\alpha_{n} \mu B\right)\left(y_{n}-p\right)\right\rangle \\
& \leq \alpha_{n}^{2}\left\|\left(\gamma f\left(x_{n}\right)-\mu B p\right)\right\|^{2}+\left(1-\alpha_{n} \tau\right)^{2}\left[\beta_{n}\left\|x_{n}-p\right\|^{2}+\left(1-\beta_{n}\right)\left\|\Im_{n}^{M} x_{n}-p\right\|^{2}\right] \\
& +2 \alpha_{n}\left\langle\left(\gamma f\left(x_{n}\right)-\mu B p\right),\left(I-\alpha_{n} \mu B\right)\left(y_{n}-p\right)\right\rangle \\
& \leq\left(1-\alpha_{n} \tau\right)^{2} \beta_{n}\left\|x_{n}-p\right\|^{2}+\left(1-\alpha_{n} \tau\right)^{2}\left(1-\beta_{n}\right)\left\|\Im_{\Im_{n}}^{M} x_{n}-p\right\|^{2}+c_{n} \\
& =\left(1-\alpha_{n} \tau\right)^{2} \beta_{n}\left\|x_{n}-p\right\|^{2}+\left(1-2 \alpha_{n}(\tau-\gamma)\right)\left(1-\beta_{n}\right)\left\|\Im_{n}^{M} x_{n}-p\right\|^{2} \\
& +\alpha_{n}^{2} \tau^{2}\left(1-\beta_{n}\right)\left\|\Im_{n}^{M} x_{n}-p\right\|^{2} \\
& -2 \alpha_{n} \gamma\left(1-\beta_{n}\right)\left\|\Im_{n}^{M} x_{n}-p\right\|^{2}+c_{n} \\
& \leq\left(1-\alpha_{n} \tau\right)^{2} \beta_{n}\left\|x_{n}-p\right\|^{2}+\left(1-2 \alpha_{n}(\tau-\gamma)\right)\left(1-\beta_{n}\right)\left\|\Im_{\mathfrak{S}_{n}}^{M} x_{n}-p\right\|^{2} \\
& +\alpha_{n}^{2} \tau^{2}\left(1-\beta_{n}\right)\left\|\Im_{n}^{M} x_{n}-p\right\|^{2}+c_{n} \\
& =\left(1-2 \alpha_{n}(\tau-\gamma)\right) \beta_{n}\left\|x_{n}-p\right\|^{2}+\alpha_{n}^{2} \tau^{2} \beta_{n}\left\|x_{n}-p\right\|^{2}-2 \alpha_{n} \gamma \beta_{n}\left\|x_{n}-p\right\|^{2} \\
& +\left(1-2 \alpha_{n}(\tau-\gamma)\right)\left(1-\beta_{n}\right)\left\|\Im_{n}^{k+1} x_{n}-p\right\|^{2} \\
& +\alpha_{n}^{2} \tau^{2}\left(1-\beta_{n}\right)\left\|\Im_{\Im_{n}}^{M} x_{n}-p\right\|^{2}+c_{n} \\
& =\left(1-2 \alpha_{n}(\tau-\gamma)\right) \beta_{n}\left\|x_{n}-p\right\|^{2}+\alpha_{n}^{2} \tau^{2} \beta_{n}\left\|x_{n}-p\right\|^{2}-2 \alpha_{n} \gamma \beta_{n}\left\|x_{n}-p\right\|^{2} \\
& +\left(1-2 \alpha_{n}(\tau-\gamma)\right)\left(1-\beta_{n}\right)\left[\left\|x_{n}-p\right\|^{2}-\left\|\Im_{n}^{k+1} x_{n}-\Im_{n}^{k} x_{n}\right\|^{2}\right] \\
& +\alpha_{n}^{2} \tau^{2}\left(1-\beta_{n}\right)\left\|\Im_{\mathfrak{S}_{n}}^{M} x_{n}-p\right\|^{2}+c_{n} \\
& \leq\left(1-2 \alpha_{n}(\tau-\gamma)\right)\left\|x_{n}-p\right\|^{2}+\alpha_{n}^{2} \tau^{2}\left\|x_{n}-p\right\|^{2} \\
& -\left(1-2 \alpha_{n}(\tau-\gamma)\right)\left(1-\beta_{n}\right)\left\|\Im_{n}^{k+1} x_{n}-\Im_{n}^{k} x_{n}\right\|^{2}+c_{n},
\end{aligned}
$$

where

$$
c_{n}=2 \alpha_{n}\left\langle\left(\gamma f\left(x_{n}\right)-\mu B p\right),\left(I-\alpha_{n} \mu B\right)\left(y_{n}-p\right)\right\rangle .
$$

It follows from the condition $(\mathrm{C} 1)$ that

$$
\lim _{n \rightarrow \infty} c_{n}=0
$$

So, we obtain

$$
\begin{aligned}
(1- & \left.2 \alpha_{n}(\tau-\gamma)\right)\left(1-\beta_{n}\right)\left\|\Im_{n}^{k+1} x_{n}-\Im_{n}^{k} x_{n}\right\|^{2} \\
\leq & \left(1-2 \alpha_{n}(\tau-\gamma)\right)\left\|x_{n}-p\right\|^{2}-\left\|x_{n+1}-p\right\|^{2} \\
& \quad+\alpha_{n}^{2} \tau^{2}\left\|x_{n}-p\right\|^{2}+c_{n} \\
\leq & \left\|x_{n}-p\right\|^{2}-\left\|x_{n+1}-p\right\|^{2}+\alpha_{n}^{2} \tau^{2}\left\|x_{n}-p\right\|^{2}+c_{n} \\
= & \left\|x_{n}-x_{n+1}\right\|\left(\left\|x_{n}-p\right\|-\left\|x_{n+1}-p\right\|\right)+\alpha_{n}^{2} \tau^{2}\left\|x_{n}-p\right\|^{2}+c_{n} .
\end{aligned}
$$


Using the condition (C1), (3.17) and (3.22), we obtain

$$
\lim _{n \rightarrow \infty}\left\|\Im_{n}^{k+1} x_{n}-\Im_{n}^{k} x_{n}\right\|=0, \quad \forall k=1,2, \ldots, M-1
$$

Step 6. We show that $\limsup _{n \rightarrow \infty}\left\langle(\gamma f-\mu B) q, x_{n}-q\right\rangle \leq 0$, where $q=P_{\Theta}(I-\mu B+\gamma f) q$.

The Banach contraction principle guarantees that $P_{\Theta}(I-\mu B+\gamma f)$ has a unique fixed point $q$ which is the unique solution of (3.1). Let $\left\{x_{n_{k}}\right\}$ be a subsequence of $\left\{x_{n}\right\}$ such that

$$
\lim _{k \rightarrow \infty}\left\langle(\gamma f-\mu B) q, x_{n_{m}}-q\right\rangle=\lim \sup _{n \rightarrow \infty}\left\langle(\gamma f-\mu B) q, x_{n}-q\right\rangle .
$$

Since $\left\{x_{n_{k}}\right\}$ is bounded, then there exists a subsequence $\left\{x_{n_{k}}\right\}$ which converges weakly to $z \in H$. Without loss of generality, we can assume that $x_{n_{k}} \rightarrow z$. We claim that $z \in \Theta$.

Next, we need to show that $z \in \bigcap_{j=1}^{M} S E P\left(F_{j}\right)$. First, by (A2) and given $y \in C$ and $k \in$ $\{1,2, \ldots, M-1\}$, we have

$$
\frac{1}{r_{k+1, n}}\left\langle y-\Im_{n}^{k+1} x_{n}, \Im_{n}^{k+1} x_{n}-\Im_{n}^{k} x_{n}\right\rangle \geq F_{k+1}\left(y, \Im_{n}^{k+1} x_{n}\right) .
$$

Thus,

$$
\left\langle y-\Im_{n_{m}}^{k+1} x_{n_{m}}, \frac{\Im_{n_{m}}^{k+1} x_{n_{m}}-\Im_{n_{m}}^{k} x_{n_{m}}}{r_{k+1, n_{m}}}\right\rangle \geq F_{k+1}\left(y, \Im_{n_{m}}^{k+1} x_{n_{m}}\right) .
$$

From (A4), $F(y, \cdot)$ is a lower semicontinuous and convex, and thus weakly semicontinuous. The condition (C3) and (3.23) imply that

$$
\frac{\Im_{n_{m}}^{k+1} x_{n_{m}}-\Im_{n_{m}}^{k} x_{n_{m}}}{r_{k+1, n_{m}}} \rightarrow 0,
$$

in norm. Therefore, letting $m \rightarrow \infty$ in (3.24) yields

$$
F_{k+1}(y, z) \leq \lim _{m \rightarrow \infty} F_{k+1}\left(y, \Im_{n_{m}}^{k+1} x_{n_{m}}\right) \leq 0,
$$

for all $y \in H$ and $k \in\{1,2, \ldots, M-1\}$. Replacing $y$ with $y_{t}=t y+(1-t) z$ with $t \in(0,1)$ and using (A1) and (A4), we obtain

$$
0=F_{k+1}\left(y_{t}, y_{t}\right) \leq t F_{k+1}\left(y_{t}, y\right)+(1-t) F_{k+1}\left(y_{t}, z\right) \leq t F_{k+1}\left(y_{t}, y\right) .
$$

Hence, $F_{k+1}(t y+(1-t) z, y) \geq 0$, for all $t \in(0,1)$ and $y \in H$. Letting $t \rightarrow 0^{+}$and using (A3), we conclude that $F_{k+1}(z, y) \geq 0$ for all $y \in H$ and $k \in\{1,2, \ldots, M\}$. Therefore,

$$
z \in E P\left(F_{j}\right), \quad \forall j=1,2, \ldots, M
$$

that is,

$$
z \in \bigcap_{j=1}^{M} \operatorname{SEP}\left(F_{j}\right) .
$$


Next, we show that $z \in \bigcap_{i=1}^{\infty} F\left(T_{i}\right)$. By Lemma 2.6, we have

$$
W_{n_{m}} z \rightarrow W z, \quad \forall z \in C
$$

and $F(W)=\bigcap_{i=1}^{\infty} F\left(T_{i}\right)$. Assume that $z \notin F(W)$, then $z \neq W z$. Therefore, from the Opial property of a Hilbert space, (3.27), (3.28) and Step 4, we have

$$
\begin{aligned}
\liminf _{m \rightarrow \infty}\left\|x_{n_{m}}-z\right\|< & \liminf _{m \rightarrow \infty}\left\|x_{n_{m}}-W z\right\| \\
\leq & \liminf _{m \rightarrow \infty}\left\{\left\|x_{n_{m}}-W_{n_{m}} \Im_{n_{m}}^{M} x_{n_{m}}\right\|\right. \\
& \left.\quad+\left\|W_{n_{m}} \Im_{n_{m}}^{M} x_{n_{m}}-W_{n_{m}} \Im_{n_{m}}^{M} z\right\|+\left\|W_{n_{m}} \Im_{n_{m}}^{M} z-W z\right\|\right\} \\
\leq & \liminf _{m \rightarrow \infty}\left\{\left\|x_{n_{m}}-z\right\|+\left\|W_{n_{m}} z-W z\right\|\right\} \\
\leq & \liminf _{m \rightarrow \infty}\left\|x_{n_{m}}-z\right\| .
\end{aligned}
$$

It is a contradiction. Thus $z$ belongs to $F(W)=\bigcap_{i=1}^{\infty} F\left(T_{i}\right)$. Hence, $z \in \Theta$.

Hence, by Lemma 2.4, we obtain

$$
\begin{aligned}
\lim \sup _{n \rightarrow \infty}\left\langle(\gamma f-\mu B) q, x_{n}-q\right\rangle & =\lim \sup _{m \rightarrow \infty}\left\langle(\gamma f-\mu B) q, x_{n_{m}}-q\right\rangle \\
& =\langle(\gamma f-\mu B) q, z-q\rangle \leq 0 .
\end{aligned}
$$

Step 7. We claim that $x_{n}$ converges strongly to $q=P_{\Theta}(I-\mu B+\gamma f) q$. We observe that

$$
\begin{aligned}
\left\|x_{n+1}-q\right\|^{2}= & \left\|\alpha_{n} \gamma f\left(x_{n}\right)+\left(I-\mu \alpha_{n} B\right) y_{n}-q\right\|^{2} \\
= & \left\|\alpha_{n} \gamma f\left(x_{n}\right)+\left(I-\mu \alpha_{n} B\right) y_{n}-q+\mu \alpha_{n} B q-\mu \alpha_{n} F q\right\|^{2} \\
\leq & \left\|\left(I-\mu \alpha_{n} B\right) y_{n}-\left(I-\mu \alpha_{n} B\right) q\right\|^{2}+2 \alpha_{n}\left\langle\gamma f\left(x_{n}\right)-\mu B q, x_{n+1}-q\right\rangle \\
\leq & \left(1-\alpha_{n} \tau\right)^{2}\left\|y_{n}-q\right\|^{2} \\
& +2 \alpha_{n}\left\langle\gamma f\left(x_{n}\right)-\gamma f(q), x_{n+1}-q\right\rangle+2 \alpha_{n}\left\langle\gamma f(q)-\mu B q, x_{n+1}-q\right\rangle \\
\leq & \left(1-\alpha_{n} \tau\right)^{2}\left\|x_{n}-q\right\|^{2}+\alpha_{n} \gamma \alpha\left(\left\|x_{n}-q\right\|^{2}+\left\|x_{n+1}-q\right\|^{2}\right) \\
& +2 \alpha_{n}\left\langle\gamma f(q)-\mu B q, x_{n+1}-q\right\rangle \\
& \frac{\left(1-\alpha_{n} \tau\right)^{2}}{1-\alpha_{n} \gamma \alpha}\left\|x_{n}-q\right\|^{2}+\frac{\alpha_{n} \gamma \alpha}{1-\alpha_{n} \gamma \alpha}\left\|x_{n}-q\right\|^{2} \\
& +\frac{2 \alpha_{n}}{1-\alpha_{n} \gamma \alpha}\left\langle\gamma f(q)-\mu B q, x_{n+1}-q\right\rangle \\
& \left.+\frac{\alpha_{n} \tau^{2}}{2(\tau-\gamma \alpha)}\left\|x_{n}-q\right\|^{2}\right) \\
& \left(1-\frac{2 \alpha_{n}(\tau-\gamma \alpha)+\alpha_{n}^{2} \tau^{2}}{1-\alpha_{n} \gamma \alpha}\right)\left\|x_{n}-q\right\|^{2}+\frac{2 \alpha_{n}}{1-\alpha_{n} \gamma \alpha}\left\langle\gamma f(q)-\mu B q, x_{n+1}-q\right\rangle \\
= & \left(1-\frac{2 \alpha_{n}(\tau-\gamma \alpha)}{1-\alpha_{n} \gamma \alpha}\right)\left\|x_{n}-q\right\|^{2} \\
& +\tau-\gamma \alpha) \\
& \frac{1}{(\tau-\gamma \alpha)}\left\langle\gamma f(q)-\mu B q, x_{n+1}-q\right\rangle \\
& \\
& \left(1-\alpha_{n} \gamma \alpha\right. \\
&
\end{aligned}
$$




$$
\begin{aligned}
= & \left(1-\frac{2 \alpha_{n}(\tau-\gamma \alpha)}{1-\alpha_{n} \gamma \alpha}\right)\left\|x_{n}-q\right\|^{2} \\
& +\frac{2 \alpha_{n}(\tau-\gamma \alpha)}{1-\alpha_{n} \gamma \alpha}\left(\frac{1}{(\tau-\gamma \alpha)}\left\langle\gamma f(q)-\mu B q, x_{n+1}-q\right\rangle+\frac{\alpha_{n} \tau^{2}}{2(\tau-\gamma \alpha)} M_{3}\right),
\end{aligned}
$$

where $M_{3}=\sup _{n \geq 1}\left\|x_{n}-q\right\|^{2}$. Put $\lambda_{n}=\frac{2 \alpha_{n}(\tau-\gamma \alpha)}{1-\alpha_{n} \gamma \alpha}$ and $\delta_{n}=\frac{1}{\tau-\gamma \alpha}\left\langle\gamma f(q)-\mu B q, x_{n+1}-q\right\rangle$. It follows that

$$
\left\|x_{n+1}-q\right\|^{2} \leq\left(1-\lambda_{n}\right)\left\|x_{n}-q\right\|^{2}+\lambda_{n} \delta_{n}+\gamma_{n}
$$

From (C1), (C2) and Step 5, it follows that $\sum_{n=0}^{\infty} \lambda_{n}=\infty$ and lim $\sup _{n \rightarrow \infty} \delta_{n} \leq 0$. Hence, by Lemma 2.7 , the sequence $\left\{x_{n}\right\}$ converges strongly to $q$.

Using Theorem 3.1, we obtain the following corollaries.

Corollary 3.2 Let $C$ be a nonempty closed convex subset of a real Hilbert space H. Let $\left\{T_{i}\right\}$ be an infinite family of nonexpansive mappings with $\bigcap_{i=1}^{\infty} F\left(T_{i}\right) \neq \emptyset, \mathcal{F}=\left\{F_{j}: j=\right.$ $1,2,3, \ldots, M\}$ be a finite family of bifunctions $C \times C$ into $\mathbb{R}$ satisfying (A1)-(A4) and $\gamma_{i}$ be a real sequence such that $0 \leq \gamma_{i} \leq b \leq 1$ for each $i \geq 1$. Let $B$ be a $k$-Lipschitzian and $\eta$ strongly monotone operator on $C$ with $0<\mu<\eta / k^{2}$ and $f \in \Pi_{H}$ with $0<\gamma<\mu\left(\eta-\frac{\mu k^{2}}{2}\right) / \alpha=$ $\tau / \alpha$ and $\tau<1$. Assume that $\Theta:=\left(\bigcap_{i=1}^{\infty} F\left(T_{i}\right)\right) \cap\left(\bigcap_{j=1}^{M} S E P\left(F_{j}\right)\right) \neq \emptyset$. Let the mapping $W_{n}$ be defined by (2.3). Let $\left\{x_{n}\right\}$ be the sequence generated by $x_{1} \in H$ and

$$
\left\{\begin{array}{l}
y_{n}=\beta_{n} x_{n}+\left(1-\beta_{n}\right) W_{n} J_{r_{M, n}}^{F_{M}} J_{r_{M-1, n}}^{F_{M-1}} \cdots J_{r_{2, n}}^{F_{2}} J_{r_{1, N}}^{F_{1}} x_{n}, \\
x_{n+1}=\alpha_{n} \gamma f\left(x_{n}\right)+\left(I-\alpha_{n} \mu B\right) y_{n}, \quad \forall n \in \mathbb{N} \text { and } n \geq 1,
\end{array}\right.
$$

where $\left\{\alpha_{n}\right\}$ and $\left\{\beta_{n}\right\}$ are the sequences in $(0,1)$ which satisfy the following conditions:

(C1) $\lim _{n \rightarrow \infty} \alpha_{n}=0$ and $\Sigma_{n=1}^{\infty} \alpha_{n}=\infty$;

(C2) $0<\liminf _{n \rightarrow \infty} \beta_{n}<\lim \sup _{n \rightarrow \infty} \beta_{n} \leq a<1$ for some constant $a \in(0,1)$;

(C3) $\liminf _{n \rightarrow \infty} r_{j, n}>0$, for each $j=1,2, \ldots, M$.

Then the sequence $\left\{x_{n}\right\}$ converges strongly to $q \in \Theta$ where $q=P_{\Theta}(I-\mu B+\gamma f) q$, which is the unique solution of the variational inequality

$$
\langle(\gamma f-\mu B) q, p-q\rangle \leq 0, \quad \forall p \in \Theta .
$$

Remark 3.3 Corollary 3.2 extends and improves Theorem 3.1 from $f$ an infinite family of nonexpansive mappings to a family of strictly pseudo contractive mappings.

If $M=1$ in Theorem 3.1, we obtain the following corollary.

Corollary 3.4 Let $C$ be a nonempty closed convex subset of a real Hilbert space H. Let $\left\{T_{i}\right\}$ be an infinite family of nonexpansive mappings with $\bigcap_{i=1}^{\infty} F\left(T_{i}\right) \neq \emptyset, \mathcal{F}=\left\{F_{j}: j=\right.$ $1,2,3, \ldots, M\}$ be a finite family of bifunctions $C \times C$ into $\mathbb{R}$ satisfying (A1)-(A4) and $\gamma_{i}$ be a real sequence such that $0 \leq \gamma_{i} \leq b \leq 1$ for each $i \geq 1$. Let $B$ be a $k$-Lipschitzian and $\eta$ strongly monotone operator on $C$ with $0<\mu<\eta / k^{2}$ and $f \in \Pi_{H}$ with $0<\gamma<\mu\left(\eta-\frac{\mu k^{2}}{2}\right) / \alpha=$ $\tau / \alpha$ and $\tau<1$. Assume that $\Theta:=\left(\bigcap_{i=1}^{\infty} F\left(T_{i}\right)\right) \cap\left(\bigcap_{j=1}^{M} S E P\left(F_{j}\right)\right) \neq \emptyset$. Let the mapping $W_{n}$ be 
defined by (2.3). Let $\left\{x_{n}\right\}$ be the sequence generated by $x_{1} \in H$ and

$$
\left\{\begin{array}{l}
F\left(u_{n}, y\right)+\frac{1}{r}\left\langle y-u_{n}, u_{n}-x_{n}\right\rangle \geq 0, \quad \forall y \in C, \\
y_{n}=\beta_{n} x_{n}+\left(1-\beta_{n}\right) W_{n} u_{n}, \\
x_{n+1}=\alpha_{n} \gamma f\left(x_{n}\right)+\left(I-\alpha_{n} \mu B\right) y_{n}, \quad \forall n \in \mathbb{N} \text { and } n \geq 1,
\end{array}\right.
$$

where $\left\{\alpha_{n}\right\}$ and $\left\{\beta_{n}\right\}$ are the sequences in $(0,1)$ which satisfy the following conditions:

(C1) $\lim _{n \rightarrow \infty} \alpha_{n}=0$ and $\Sigma_{n=1}^{\infty} \alpha_{n}=\infty$;

(C2) $0<\liminf _{n \rightarrow \infty} \beta_{n}<\lim \sup _{n \rightarrow \infty} \beta_{n} \leq a<1$ for some constant $a \in(0,1)$;

(C3) $\liminf _{n \rightarrow \infty} r_{j, n}>0$, for each $j=1,2, \ldots, M$.

Then the sequence $\left\{x_{n}\right\}$ converges strongly to $q \in \Theta$, where $q=P_{\Theta}(I-\mu B+\gamma f) q$, which is the unique solution of the variational inequality

$$
\langle(\gamma f-\mu B) q, p-q\rangle \leq 0, \quad \forall p \in \Theta .
$$

If $M=1, W_{n}=W, \gamma=1, A=I$ and $\mu=1$ in Theorem 3.1, we obtain the following corollary:

Corollary 3.5 Let $C$ be a nonempty closed convex subset of a real Hilbert space $H$. Let $\left\{T_{i}\right\}$ be an infinite family of nonexpansive mappings with $\bigcap_{i=1}^{\infty} F\left(T_{i}\right) \neq \emptyset, \mathcal{F}=\left\{F_{j}: j=\right.$ $1,2,3, \ldots, M\}$ be a finite family of bifunctions $C \times C$ into $\mathbb{R}$ satisfying (A1)-(A4) and $\gamma_{i}$ be a real sequence such that $0 \leq \gamma_{i} \leq b \leq 1$ for each $i \geq 1$. Let $B$ be a $k$-Lipschitzian and $\eta$ strongly monotone operator on $C$ with $0<\mu<\eta / k^{2}$ and $f \in \Pi_{H}$ with $0<\gamma<\mu\left(\eta-\frac{\mu k^{2}}{2}\right) / \alpha=$ $\tau / \alpha$ and $\tau<1$. Assume that $\Theta:=\left(\bigcap_{i=1}^{\infty} F\left(T_{i}\right)\right) \cap\left(\bigcap_{j=1}^{M} S E P\left(F_{j}\right)\right) \neq \emptyset$. Let the mapping $W_{n}$ be defined by (2.3). Let $\left\{x_{n}\right\}$ be the sequence generated by $x_{1} \in H$ and

$$
\left\{\begin{array}{l}
y_{n}=\beta_{n} x_{n}+\left(1-\beta_{n}\right) W J_{r_{M, n}}^{F_{M}} J_{r_{M-1, n}}^{F_{M-1}} \cdots J_{r_{2, n}}^{F_{2}} J_{r_{1, N}}^{F_{1}} x_{n}, \\
x_{n+1}=\alpha_{n} f\left(x_{n}\right)+\left(I-\alpha_{n} \mu B\right) y_{n}, \quad \forall n \in \mathbb{N} \text { and } n \geq 1,
\end{array}\right.
$$

where $\left\{\alpha_{n}\right\}$ and $\left\{\beta_{n}\right\}$ are the sequences in $(0,1)$ which satisfy the following conditions:

(C1) $\lim _{n \rightarrow \infty} \alpha_{n}=0$ and $\Sigma_{n=1}^{\infty} \alpha_{n}=\infty$;

(C2) $0<\liminf _{n \rightarrow \infty} \beta_{n}<\limsup \sup _{n \rightarrow \infty} \beta_{n} \leq a<1$ for some constant $a \in(0,1)$;

(C3) $\liminf _{n \rightarrow \infty} r_{j, n}>0$, for each $j=1,2, \ldots, M$.

Then the sequence $\left\{x_{n}\right\}$ converges strongly to $q \in \Theta$ where $q=P_{\Theta}(I-\mu B+\gamma f) q$, which is the unique solution of the variational inequality

$$
\langle(\gamma f-\mu B) q, p-q\rangle \leq 0, \quad \forall p \in \Theta
$$

\section{Numerical example}

In this section, we give a real numerical example of Theorem 3.1 as follows.

Example 4.1 Let $H=\mathbb{R}, C=\left[0, \frac{1}{4}\right], T_{n}=I . F_{k}(x, y)=0, \forall x, y \in H, r_{k, n}=1, k \in\{1,2,3, \ldots, K\}$, $B=I, f(x)=x^{2}, \beta_{n}=\frac{1}{2}, \alpha_{n}=\frac{1}{n}$ for every $n \in \mathbb{N}$ and $\mu=1$. Then $\left\{x_{n}\right\}$ is the sequence generated by

$$
x_{n+1}=\frac{x_{n}^{2}}{n}+\left(1-\frac{1}{n}\right) x_{n},
$$


and $z \rightarrow 0$ as $n \rightarrow \infty$, where 0 is the unique solution of the minimization problem

$$
\min _{x \in C} \frac{x^{3}}{3}+\frac{x^{2}}{2}+C_{1}
$$

where $C_{1}$ is a constant.

Proof We divide the proof into four steps.

Step 1. Using the idea in [7], we can show that

$$
J_{r_{k, n}}^{k} x=P_{C} x, \quad \forall x \in H, k \in\{1,2, \ldots, K\}
$$

where

$$
P_{C} x= \begin{cases}\frac{x}{|x|}, & x \in H-C, \\ x, & x \in C .\end{cases}
$$

Since $F_{k}(x, y)=0, \forall x, y \in C, k \in\{1,2, \ldots, K\}$, with the definition of $J_{r}(x), \forall x \in H$ in Lemma 2.13, we have

$$
J_{r}^{F}(x)=\left\{z \in C: F(z, y)+\frac{1}{r}\langle y-z, z-x\rangle \geq 0, \forall y \in C\right\} .
$$

By the equivalent property of the nearest projection $P_{C}$ from $H$ to $C$, we can conclude that if we take $x \in C, J_{r_{k, n}}^{k} x=P_{C} x=I x$. By (3) in Lemma 2.13, we have

$$
\bigcap_{k=1}^{K} \operatorname{SEP}\left(F_{k}\right)=C
$$

Step 2. We show that

$$
W_{n}=I .
$$

Since $T_{i}^{\prime}=\theta_{i} I+\left(1-\theta_{i}\right) T_{i}$, where $T_{i}$ is a $\lambda_{i}$-strictly pseudo-contractive mapping and $\theta_{i} \in$ $\left[\lambda_{i}, 1\right)$, it can be easily seen that $T_{i}^{\prime}$ is a nonexpansive mapping. By (2.3), we have

$$
\begin{aligned}
& W_{1}=U_{1,1}=\gamma_{1} T_{1}^{\prime} U_{1,2}+\left(1-\gamma_{1}\right) I, \\
& \begin{aligned}
W_{2}=U_{2,1} & =\gamma_{1} T_{1}^{\prime} U_{2,2}+\left(1-\gamma_{1}\right) I=\gamma_{1} T_{1}^{\prime}\left(\gamma_{2} T_{2}^{\prime} U_{2,3}+\left(1-\gamma_{2}\right) I\right)+\left(1-\gamma_{1}\right) I \\
& =\gamma_{1} \gamma_{2} T_{1}^{\prime} T_{2}^{\prime}+\gamma_{1}\left(1-\gamma_{2}\right) T_{1}^{\prime}+\left(1-\gamma_{1}\right) I, \\
W_{3}=U_{3,1} & =\gamma_{1} T_{1}^{\prime} U_{3,2}+\left(1-\gamma_{1}\right) I=\gamma_{1} T_{1}^{\prime}\left(\gamma_{2} T_{2}^{\prime} U_{3,3}+\left(1-\gamma_{2}\right) I\right)+\left(1-\gamma_{1}\right) I \\
& =\gamma_{1} \gamma_{2} T_{1}^{\prime} T_{2}^{\prime} U_{3,3}+\gamma_{1}\left(1-\gamma_{2}\right) T_{1}^{\prime}+\left(1-\gamma_{1}\right) I \\
& =\gamma_{1} \gamma_{2} T_{1}^{\prime} T_{2}^{\prime}\left(\gamma_{3} T_{3}^{\prime} U_{3,4}+\left(1-\gamma_{3}\right) I\right)+\gamma_{1}\left(1-\gamma_{2}\right) T_{1}^{\prime}+\left(1-\gamma_{1}\right) I \\
& =\gamma_{1} \gamma_{2} \gamma_{3} T_{1}^{\prime} T_{2}^{\prime} T_{3}^{\prime}+\gamma_{1} \gamma_{2}\left(1-\gamma_{3}\right) T_{1}^{\prime} T_{2}^{\prime}+\gamma_{1}\left(1-\gamma_{2}\right) T_{1}^{\prime}+\left(1-\gamma_{1}\right) I,
\end{aligned}
\end{aligned}
$$


and we compute (2.3) in the same way as above, so we obtain

$$
\begin{aligned}
W_{n}= & U_{n, 1}=\gamma_{1} \gamma_{2} \cdots \gamma_{n} T_{1}^{\prime} T_{2}^{\prime} \cdots T_{n}^{\prime}+\gamma_{1} \gamma_{2} \cdots \gamma_{n-1}\left(1-\gamma_{n}\right) T_{1}^{\prime} T_{2}^{\prime} \cdots T_{n-1}^{\prime} \\
& +\gamma_{1} \gamma_{2} \cdots \gamma_{n-2}\left(1-\gamma_{n-1}\right) T_{1}^{\prime} T_{2}^{\prime} \cdots T_{n-2}^{\prime}+\cdots+\gamma_{1}\left(1-\gamma_{2}\right) T_{1}^{\prime}+\left(1-\gamma_{1}\right) I .
\end{aligned}
$$

Since $T_{n}^{\prime}=I, \gamma_{n}=\beta, n \in \mathbb{N}$, hence,

$$
W_{n}=\left[\beta^{n}+\beta^{n-1}(1-\beta)+\cdots+\beta(1-\beta)+(1-\beta)\right] I=I
$$

Step 3. We prove

$$
x_{n+1}=\frac{x_{n}^{2}}{n}+\left(1-\frac{1}{n}\right) x_{n} \quad \text { and } \quad x_{n} \rightarrow 0 \quad \text { as } n \rightarrow \infty,
$$

where 0 is the unique solution of the minimization problem

$$
\min _{x \in C} \frac{x^{3}}{3}+\frac{x^{2}}{2}+C_{1}
$$

Since we let $B=I, \gamma$ is a real number, so we choose $\gamma=1$. From (4.3), (4.4) and (4.7), we can obtain a special sequence $\left\{x_{n}\right\}$ of Theorem 3.1 as follows:

$$
x_{n+1}=\frac{x_{n}^{2}}{n}+\left(1-\frac{1}{n}\right) x_{n} .
$$

Since $T_{n}=I, n \in \mathbb{N}$, we have

$$
\bigcap_{n \in \mathbb{N}} F\left(T_{n}\right)=H
$$

Combining it with (4.6), we obtain

$$
\Theta:=\left(\bigcap_{k=1}^{K} \operatorname{SEP}\left(F_{k}\right)\right) \cap\left(\bigcap_{n \in \mathbb{N} F\left(T_{n}\right)}\right)=C=\left[0, \frac{1}{4}\right] .
$$

It is obvious that $x_{n} \rightarrow 0,0$ is the unique solution of the minimization problem $\min _{x \in C} \frac{x^{3}}{3}+$ $\frac{x^{2}}{2}+C_{1}$, where $C_{1}$ is a constant number.

Step 4. In this step, we give the numerical results that support our main theorem as shown by plotting graphs using Matlab 7.11.0. We choose two different initial values as $x_{1}=0.1$ and $x_{1}=0.15$ in Table 1, Figure 1, and Figure 2, respectively. From the example, we can see that $\left\{x_{n}\right\}$ converges to 0 . 
Table 1 The sequence values on each different iteration step

\begin{tabular}{llllll}
\hline Iteration step $(\mathrm{n})$ & $x(1)=0.1$ & $x(1)=0.15$ & Iteration step $(\mathrm{n})$ & $x(1)=0.1$ & $x(1)=0.15$ \\
\hline 0 & 0.1000 & 0.1500 & 7 & 0.0015 & 0.0033 \\
1 & 0.0100 & 0.0225 & 8 & 0.0013 & 0.0029 \\
2 & 0.0051 & 0.0115 & 9 & 0.0011 & 0.0026 \\
3 & 0.0034 & 0.0077 & 203 & 0.0001 & 0.0001 \\
4 & 0.0025 & 0.0058 & 204 & 0.0000 & 0.0001 \\
5 & 0.0020 & 0.0046 & 205 & 0.0000 & 0.0001 \\
6 & 0.0017 & 0.0039 & 250 & 0.0000 & 0.0000 \\
\hline
\end{tabular}

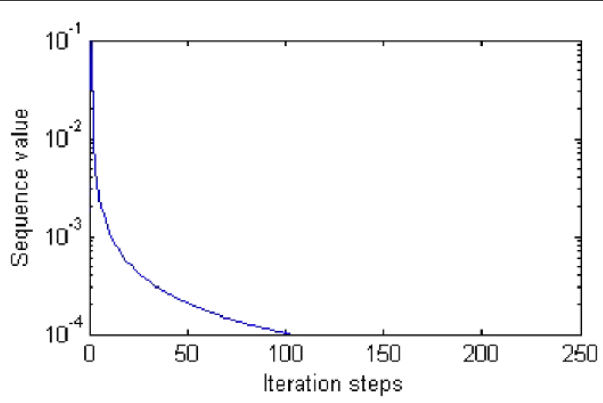

Figure 1 The initial value $x(1)=0.1$ and iteration steps $n=250$.

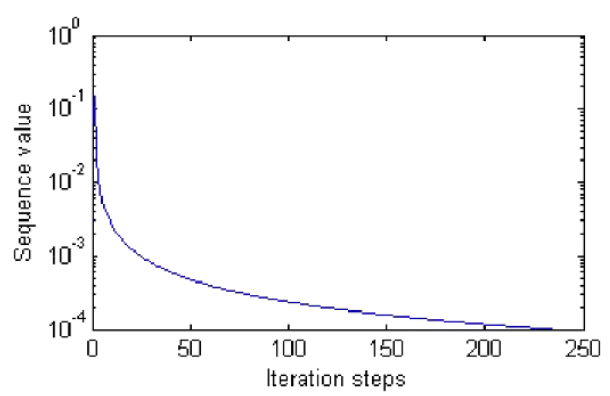

Figure 2 The initial value $x(1)=0.15$ and iteration steps $n=250$

\section{Author details}

'Department of Mathematics, Faculty of Science, King Mongkut's University of Technology Thonburi, KMUTT, Bangkok, 10140, Thailand. 'Department of Mathematics Education, Kyungnam University, Masan, 631-701, Korea.

\section{Acknowledgements}

This research was partially finished during the last visit at Kyungnam University, South Korea. The first author would like to thank the Office of the Higher Education Commission, Thailand, for the financial support of the PhD Program at KMUTT. The third author would like to thank the Higher Education Research Promotion and National Research University Project of Thailand, Office of the Higher Education Commission (under the project NRU-CSEC No.55000613) for financial support during the preparation of this manuscript. Finally, the authors would like to thank the referees for reading this paper carefully, providing valuable suggestions and comments, and improving the original version of this paper.

Received: 26 July 2012 Accepted: 21 September 2012 Published: 9 October 2012

\section{References}

1. Marino, G, Xu, HK: A general iterative method for nonexpansive mappings in Hilbert spaces. J. Math. Anal. Appl. 318, 43-52 (2006)

2. Jung, JS: Strong convergence of iterative methods for $k$-strictly pseudo-contractive mappings in Hilbert spaces. Appl. Math. Comput. 215, 3746-3753 (2010)

3. Tian, M: A general iterative algorithm for nonexpansive mappings in Hilbert spaces. Nonlinear Anal. 73, 689-694 (2010)

4. Saeidi, S: Modified hybrid steepest-descent methods for variational inequalities and fixed points. Math. Comput. Model. 52, 134-142(2010) 
5. Shimoji, K, Takahashi, W: Strong convergence to common fixed points of infinite nonexpansive mappings and applications. Taiwan. J. Math. 5(2), 387-404 (2001)

6. Wang, S: A general iterative method for obtaining an infinite family of strictly pseudo-contractive mappings in Hilbert spaces. Appl. Math. Lett. 24, 901-907 (2011)

7. He, H, Liu, S, Cho, YJ: An explicit method for systems of equilibrium problems and fixed points of infinite family of nonexpansive mappings. J. Comput. Appl. Math. (2011). doi:10.1016/j.cam.2011.03.003

8. Goebel, K, Kirk, WA: Topics on Metric Fixed-Point Theory. Cambridge University Press, Cambridge (1990)

9. Suzuki, T: Strong convergence of Krasnoselskii and Mann's type sequences for one parameter nonexpansive semigroups without Bochner integral. J. Math. Anal. Appl. 35, 227-239 (2005)

10. Liu, LS: Iterative processes with errors for nonlinear strongly accretive mappings in Banach spaces. J. Math. Anal. Appl. $194,114-125$ (1995)

11. Xu, HK: Iterative algorithms for nonlinear operators. J. Lond. Math. Soc. 66, 240-256 (2002)

12. Zhou, Y: Convergence theorems of fixed points for $k$-strict pseudo-contractions in Hilbert spaces. Nonlinear Anal. 69, 456-462 (2008)

13. Chang, S-S: A new method for solving equilibrium problem and variational inequality problem with application to optimization. Nonlinear Anal. 70, 3307-3319 (2009)

14. Colao, V, Acedob, GL, Marinoa, G: An implicit method for finding common solution of variational inequalities and systems of equilibrium problems and fixed points of infinite family of nonexpansive mappings. Nonlinear Anal. 71, 2708-2713 (2009)

15. Combettes, PL, Hirstaoga, SA: Equilibrium programming in Hilbert spaces. J. Nonlinear Convex Anal. 6, 117-136 (2005)

16. Colao, V, Marino, G, Xu, HK: An iterative method for finding common solutions of equilibrium and fixed point problems. J. Math. Anal. Appl. 344, 340-352 (2008)

doi:10.1186/1029-242X-2012-224

Cite this article as: Witthayarat et al.: A viscosity hybrid steepest-descent method for a system of equilibrium and fixed point problems for an infinite family of strictly pseudo-contractive mappings. Journal of Inequalities and Applications 2012 2012:224.

\section{Submit your manuscript to a SpringerOpen ${ }^{\ominus}$ journal and benefit from:}

- Convenient online submission

Rigorous peer review

- Immediate publication on acceptance

- Open access: articles freely available online

- High visibility within the field

- Retaining the copyright to your article 